\title{
Stereotactic radiosurgery combined with targeted/ immunotherapy in patients with melanoma brain metastasis
}

Indrawati Hadi ${ }^{* *}$, Olarn Roengvoraphoj ${ }^{1}$, Raphael Bodensohn¹, Jan Hofmaier ${ }^{1}$, Maximilian Niyazi ${ }^{1,2}$, Claus Belka, ${ }^{1,2}$ and Silke Birgit Nachbichler ${ }^{1}$

\begin{abstract}
Background: There is limited data on the use of targeted or immunotherapy (TT/TT) in combination with single fraction stereotactic radiosurgery (SRS) in patients with melanoma brain metastasis (MBM). Therefore, we analyzed the outcome and toxicity of SRS alone compared to SRS in combination with TT/T.

Methods: Patients with MBM treated with single session SRS at our department between 2014 and 2017 with a minimum follow-up of 3 months after first SRS were included. The primary endpoint of this study was local control (LC). Secondary endpoints were distant intracranial control, radiation necrosis-free survival (RNFS), and overall survival (OS). The local/ distant intracranial control rates, RNFS and OS were analyzed using the Kaplan-Meier method. The log-rank test was used to test differences between groups. Cox proportional hazard model was performed for univariate continuous variables and multivariate analyses.
\end{abstract}

Results: Twenty-eight patients (17 male and 11 female) with 52 SRS-lesions were included. The median follow-up was 19 months (range 14-24 months) after first SRS. Thirty-six lesions (69.2\%) were treated with TT/IT simultaneously (4 weeks before and 4 weeks after SRS), while 16 lesions (30.8\%) were treated with SRS alone or with sequential T/ IT. The 1-year local control rate was 100 and $83.3 \%$ for SRS with TT/IT and SRS alone $(p=0.023)$, respectively. The estimated 1-year RNFS was 90.0 and $82.1 \%$ for SRS in combination with TT/T and SRS alone ( $p=0.935)$. The distant intracranial control rate after 1 year was 47.7 and 50\% for SRS in combination with TT/IT and SRS alone ( $p=0.933)$. On univariate analysis, the diagnosis-specific Graded Prognostic Assessment including the BRAF status (MelanomamolGPA) was associated with a significantly improved LC. Neither gender nor SRS-PTV margin had a prognostic impact on LC. V10 and V12 were significantly associated with RNFS $(p<0.001$ and $p=0.004)$.

Conclusion: SRS with simultaneous TT/T significantly improved LC with no significant difference in radiation necrosis rate. The therapy combination appears to be effective and safe. However, prospective studies on SRS with simultaneous $T / / T$ are necessary and ongoing.

Trial registration: The institutional review board approved this analysis on 10th of February 2015 and all patients signed informed consent (UE nr. 128-14).

Keywords: Brain metastasis, Immunotherapy, Melanoma, Stereotactic radiosurgery

\footnotetext{
* Correspondence: Indrawati.Hadi@med.uni-muenchen.de

'Department of Radiation Oncology, University Hospital, LMU Munich,

Munich, Germany

Full list of author information is available at the end of the article
}

(c) The Author(s). 2020 Open Access This article is distributed under the terms of the Creative Commons Attribution 4.0 International License (http://creativecommons.org/licenses/by/4.0/), which permits unrestricted use, distribution, and reproduction in any medium, provided you give appropriate credit to the original author(s) and the source, provide a link to the Creative Commons license, and indicate if changes were made. The Creative Commons Public Domain Dedication waiver (http://creativecommons.org/publicdomain/zero/1.0/) applies to the data made available in this article, unless otherwise stated. 


\section{Background}

Among all malignancies, melanoma has the highest risk to spread to the brain besides lung cancer [1]. Thirtyfour percent of patients with melanoma developed brain metastases in clinical studies and $75 \%$ of melanoma patients were found to have brain metastases in autopsy studies [2]. With a median overall survival of 4.6 months brain metastases are the leading cause of death in melanoma patients [3]. Because of its resistance to radioand chemotherapy, the management of melanoma brain metastases remains challenging. Standard local treatment options for MBM are stereotactic radiosurgery (SRS), resection, or whole brain radiotherapy (WBRT). In the past, WBRT has been the standard therapy for multiple brain metastases. Nevertheless, SRS is nowadays preferred over WBRT, especially because of preserving neurocognition and because of its excellent local control rates [4]. SRS might also have synergistic effects with systemic therapy [5]. There are certain cases, that SRS could not be performed, for example due to numerous ( $>10$ lesions) and large brain metastases. In this situation, WBRT with hippocampal sparing combined with simultaneous integrated boost of the metastases enables better outcomes with less neurological toxicity $[6,7]$.

The use of targeted therapies and immune check point inhibitors, such as BRAF inhibitors, MEK inhibitors, CTLA-4 antibodies and PD-1 antibodies has changed the paradigm in metastatic melanoma patients in recent years. These systemic therapies showed to improve overall survival of patients with metastatic melanoma significantly [8].

SRS combined with immuno- or targeted therapy might be more effective than SRS alone [9]. Still, there is limited data on the effects of targeted/ immunotherapy (TT/IT) in combination with SRS as only few studies evaluated TT/IT in MBM and several studies for TT/IT excluded patients with MBM [10,11]. This study aims to analyze the outcome and toxicity of SRS alone compared with SRS and concurrent TT/IT.

\section{Patients and methods}

\section{Patients}

Patients with MBM who had been treated with single session SRS at our department between 2014 and 2017 were retrospectively identified from the institutional database. Only patients with a minimum follow-up of 3 months were included in the analysis. Patient, tumor, and treatment information were extracted from the charts. Treatment groups were categorized as SRS alone, and SRS plus concurrent TT/IT. Furthermore, the patients were assessed using the diagnosis-specific prognostic index for patients with MBM. The previous Melanoma-Graded Prognostic Assessment (MelanomaGPA) included age, Karnofsky Performance Score (KPS), the absence or presence of extracranial metastases and the number of brain metastases. BRAF status had been found to be of prognostic significance in the latest update and therefore had been incorporated as an additional factor into the new Melanoma-molGPA [12].

The institutional review board approved this analysis and all patients signed informed consent prior to the start of therapy (UE nr. 128-14).

\section{Targeted/ immunotherapy (TT/IT)}

TT/IT was considered as concurrent if it was applied within 4 weeks before or after SRS. BRAF inhibitors (dabrafenib, vemurafenib, encorafenib), MEK inhibitors (trametinib, binimetinib), CTLA-4 antibody ipilimumab, IgG4 anti-PD-1 antibody nivolumab and PD-1 antibody pembrolizumab were registered as TT/IT.

\section{SRS technique}

Single session SRS treatment planning included fusion of T1-weighted gadolinium enhanced magnetic resonance imaging (MRI) of the brain with $1 \mathrm{~mm}$ slice thickness with computed tomography (CT) simulation imaging. Patients have been immobilized in an invasive stereotactic head frame until 2014, afterwards a noninvasive thermoplastic mask system (double or Brainlab) has been used. From 2014 until 2015, a uniform $3 \mathrm{~mm}$ expansion of the GTV was used to create the PTV. This margin expansion was initially defined as our institutional standard taking into consideration positional deviation due to setup, mechanical and imaging errors during the irradiation period, and the assumed microscopic extension of the tumor around the GTV [13]. With the improvement of IGRT, GTV was expanded by $2 \mathrm{~mm}$ uniformly to create the PTV after 2015. Another margin reduction to $1 \mathrm{~mm}$ from GTV to PTV was implemented at our institution after 2017, which was not included in this analysis.

Prescription doses were determined by the treating physician based on the diameter of the metastases and the location of the lesion: metastases with a diameter of at least $4 \mathrm{~mm}$ and not exceeding $25 \mathrm{~mm}$ were treated with 18-20 Gy prescribed to the $80 \%$ isodose. Dose might be reduced because of previous irradiation, critical tumor location or irregular shape. Dose escalation to 24 Gy was allowed in uncritical small lesions. Hypofractionated stereotactic radiotherapy (SRT) was performed for metastases with diameter greater than $30 \mathrm{~mm}$, which was not included in this analysis. Radiation therapy was delivered using a linear accelerator (LINAC) with a maximum energy of $6 \mathrm{MV}$. After 2016, plans were delivered in flattening-filter-free (FFF) mode and with a maximum energy of $10 \mathrm{MV}$. Image guidance has been provided with cone beam CT and additionally with the Brainlab ExacTrac positioning system since November 2014. 


\section{Follow-up}

MRI at 2-3 months intervals as well as neurological status assessment were done to follow-up the patients after treatment. According to RECIST criteria, local failure was defined as $\mathrm{a} \geq 20 \%$ radiographic increase in the size of the previously irradiated area, which remained consistent or continued to increase on subsequent imaging [14]. Radiation necrosis was defined as a lesion with certain radiographic changes in MRI. These changes might be obvious at least 3 month post-SRS and were described as a peripheral enhancing and central necrotic lesion on T1-weighted post-gadolinium MRI sequences, as well as an increase in volume of the lesion within the high dose SRS area [15]. Subsequent metabolic imaging with 18FFluoro-Ethyl-Tyrosine positron-emission tomography (FET-PET) and stereotactic biopsy were performed to confirm the diagnosis of brain necrosis. The case of a patient with suspected RN after SRS has been discussed in the multidisciplinary tumor board with a neuroradiologist, a radiation oncologist, and a neurosurgeon to meet a consensus regarding diagnosis and treatment. RN was considered symptomatic if patients presented with neurologic symptoms, such as headache, nausea, vertigo, or other neurological deficits. Steroids or bevacizumab in case of steroid-refractory $\mathrm{RN}$ were used to treat symptomatic RN [16].

\section{Statistical analysis}

For patients with SRS for multiple lesions each lesion was analyzed independently.

The primary endpoint of this study was local BM control, which included all treated lesions not meeting the definition of local failure.

Secondary endpoints were distant intracranial control (DIC), radiation necrosis-free survival (RNFS), and overall survival (OS). DIC was defined as freedom from development of new $\mathrm{BM}$ or leptomeningeal disease outside of the irradiated volume. The local control rates were considered as the interval between the date of SRS and the date of local failure, or date of last follow up. The distant intracranial control rates were defined as the interval between the date of the first SRS and the date of brain MRI with development of new brain metastasis, or date of last follow-up. RNFS was defined as the interval between the date of SRS and the date of diagnosis of radiation necrosis, or date of last follow-up. OS was considered as the interval between the date of MRI with first diagnosis of brain metastasis and the date of death of any cause, or date of last follow-up. These endpoints were analyzed using the Kaplan-Meier method. The logrank test was used to test differences between groups. Patient demographics were calculated using descriptive statistics as absolute and relative frequencies. Cox proportional hazard model was performed for univariate continuous variables. A two tailed $p$-value of $<0.05$ was considered significant. Statistical analyses were done with IBM SPSS Statistics, Version 25 (IBM, Armonk, New York, USA).

\section{Results \\ Patient characteristics}

Thirty patients with 55 melanoma brain metastases were treated with SRS at our institution between 2014 and 2017. Patients with a follow-up shorter than 3 months were excluded, resulting in 28 patients and 52 lesions for analyses. Patients had a median age of 61 years (range 19-86 years) and developed brain metastasis with a median of 25.5 months after first diagnosis of melanoma (range $0-137$ months).

Twenty-two patients (78.6\%) had a KPS of $90-100 \%, 5$ patients $(17.9 \%)$ of $80 \%$, and one patient $(3.6 \%)$ of $70 \%$. Extracranial systemic disease was controlled in $10 \mathrm{pa}$ tients (35.7\%). BRAF-mutation (mostly V600E) was found in 14 patients (50.0\%).

Regarding TT/IT, two patients (7.1\%) were treated with BRAF inhibitors (dabrafenib or vemurafenib), one patient was treated with trametinib (3.6\%), 2 patients received ipilimumab (7.1\%), nivolumab or pembrolizumab was given in 6 patients (21.4\%). The combination of BRAFi and MEKi (dabrafenib-trametinib or encorafenibbinimetinib) was given in 3 patients (10.7\%). Nivolumabipilimumab was given in 7 patients $(25.0 \%)$. One patient (3.6\%) received a BRAFi and MEKi combination at the beginning, the therapy regimen was then changed to anti-PD-1 due to progressive disease. Another patient (3.6\%) received also a BRAFi and MEKi combination at the beginning, which was then changed to a triple combination (pembrolizumab, dabrafenib, and trametinib) due to progression. Thus, 20 patients $(71.4 \%)$ were treated with SRS and concurrent TT/IT.

Five patients $(17.9 \%)$ had no TT/IT at all, because brain metastases were diagnosed in recurrence-free and treatment-free interval. TT/IT was not applied concurrently in 3 patients.

Patients' characteristics are summarized in Table 1.

\section{Lesion characteristics and treatment parameters}

Fifty-two lesions with a median follow-up of 19 months (range 14-24 months) after SRS were analyzed. Median lesion diameter was $8 \mathrm{~mm}$ (range $4-23 \mathrm{~mm}$ ), median GTV volume $0.4 \mathrm{ccm}$ (range $0.08-6.54 \mathrm{ccm}$ ), and median PTV volume $1.6 \mathrm{ccm}$ (range $0.30-13.46 \mathrm{ccm}$ ). The most common SRS doses were $20 \mathrm{~Gy}(n=30 ; 57.7 \%)$ and 18 Gy $(n=17 ; 32.7 \%)$ prescribed at the $80 \%$ isodose line $(n=51,98.1 \%)$. One lesion was treated with SRS dose reduction to 15Gy because of previous WBRT with boost adjacent to the current lesion. 
Table 1 Patients' characteristics

\begin{tabular}{|c|c|c|}
\hline \multirow{2}{*}{$\begin{array}{l}\text { Characteristic } \\
\text { Sex }\end{array}$} & \multicolumn{2}{|c|}{ Number of patients absolute relative } \\
\hline & & \\
\hline Female & 11 & $39.3 \%$ \\
\hline Male & 17 & $60.7 \%$ \\
\hline \multicolumn{3}{|l|}{ Karnofsky performance status (KPS) } \\
\hline $90-100 \%$ & 22 & $78.6 \%$ \\
\hline $80 \%$ & 5 & $17.9 \%$ \\
\hline $70 \%$ & 1 & $3.6 \%$ \\
\hline \multicolumn{3}{|l|}{ BRAF Status } \\
\hline BRAF V600-E-Mutation & 14 & $50.0 \%$ \\
\hline No BRAF V600-E-Mutation & 14 & $50.0 \%$ \\
\hline \multicolumn{3}{|l|}{ Controlled extracranial disease } \\
\hline Yes & 10 & $35.7 \%$ \\
\hline No & 18 & $64.3 \%$ \\
\hline \multicolumn{3}{|l|}{ RPA class } \\
\hline 1 & 6 & $21.4 \%$ \\
\hline 2 & 22 & $78.6 \%$ \\
\hline 3 & 0 & $0 \%$ \\
\hline \multicolumn{3}{|l|}{ Melanoma-molGPA } \\
\hline $0.5-1.0$ & 2 & $7.1 \%$ \\
\hline $1.5-2.0$ & 11 & $39.3 \%$ \\
\hline $2.5-3.0$ & 11 & $39.3 \%$ \\
\hline $3.5-4.0$ & 4 & $14.3 \%$ \\
\hline \multicolumn{3}{|l|}{ Number of SRS lesions per patient } \\
\hline 1 & 13 & $46.4 \%$ \\
\hline 2 & 7 & $25.0 \%$ \\
\hline 3 & 7 & $25.0 \%$ \\
\hline 4 & 1 & $3.6 \%$ \\
\hline \multicolumn{3}{|l|}{ Whole brain radiotherapy } \\
\hline Yes & 4 & $14.3 \%$ \\
\hline Before first SRS & 1 & $3.6 \%$ \\
\hline After first SRS & 3 & $10.7 \%$ \\
\hline No & 24 & $85.7 \%$ \\
\hline \multicolumn{3}{|l|}{ Targeted/ immunotherapy (TT/IT) } \\
\hline $\begin{array}{l}\text { BRAFi (Dabrafenib/Vemurafenib) } \\
\text { MEKi (Trametinib) }\end{array}$ & $\begin{array}{l}2 \\
1\end{array}$ & $\begin{array}{l}7.1 \% \\
3.6 \%\end{array}$ \\
\hline Anti-CTLA-4 (Ipilimumab) & 2 & $7.1 \%$ \\
\hline Anti-PD1 (Nivolumab/Pembrolizumab) & 6 & $21.4 \%$ \\
\hline BRAFi+MEKi (Dabrafenib-Trametinib/ Encorafenib-Binimetinib) & 3 & $10.7 \%$ \\
\hline BRAFi+MEKi $\rightarrow$ Anti-PD1 & 1 & $3.6 \%$ \\
\hline Anti-PD1 + Anti-CTLA-4 (Nivolumab-Ipilimumab) & 7 & $25.0 \%$ \\
\hline BRAFi+MEKi $\rightarrow$ Triple combination (Pembrolizumab-Dabrafenib-Trametinib) & 1 & $3.6 \%$ \\
\hline none & 5 & $17.9 \%$ \\
\hline \multicolumn{3}{|l|}{ Concurrent TT/IT } \\
\hline Yes & 20 & $71.4 \%$ \\
\hline
\end{tabular}


Table 1 Patients` characteristics (Continued)

\begin{tabular}{llr}
\hline Characteristic & Number of patients absolute relative \\
\hline No & 8 & $28.6 \%$ \\
\hline
\end{tabular}

Regarding the fixation technique, an invasive stereotactic head frame was used in 5 lesions (9.6\%), a frameless Brainlab mask in 9 (17.3\%), and a frameless double thermoplastic mask in 38 (73.1\%). Treatment planning was done with Oncentra MasterPlan ${ }^{\odot}$ in 30 lesions (57.7\%), Monaco ${ }^{\circledR}$ in 17 (32.7\%), and Radionics Xplan in 5 (9.6\%).

Five lesions in 2 patients were treated with WBRT before SRS and 3 lesions in 3 patients received WBRT afterwards.

SRS only was performed in 10 lesions (19.2\%). TT/IT was applied before SRS in 24 lesions (46.2\%), the median duration from application of TT/IT to SRS was 9.5 days (range 3-43 days). TT/IT was given after SRS in 39 lesions $(75.0 \%)$ with a median duration of 9 days from SRS to application of TT/IT (2-97 days).

We defined concurrent TT/IT as a TT/IT, which was given within 4 weeks before and/or after SRS. According to this definition, TT/IT was given concurrently before SRS in 21 lesions (40.4\%, median: 8 days, range 3-21 days). Additionally, further 15 lesions received TT/IT after SRS, resulting in 36 lesions (69.2\%), which were treated with TT/IT concurrently (median: 8 days, range 2-27 days, before and/or after SRS). Lesion characteristics and treatment parameters are summarized in Table 2.

\section{Local control}

Two lesions developed local failure after SRS (3.8\%). Local control rates at 1 and 2 years for all patients with MBM treated with SRS were 98.1 and $95.4 \%$, respectively. Median local control has not been reached after a median follow-up of 14 months (range: 3-32 months) (Fig. 1a).

One-year LC rates were 100 and $83.3 \%$ for SRS with concurrent TT/IT and SRS alone $(p=0.023)$ (Fig. 2a). Furthermore, the timing of concurrent TT/IT was analyzed; it showed that, TT/IT which was given after SRS was associated with improved 1-year LC (100 and 83.3\% for concurrent TT/IT after SRS and SRS alone, $p<$ 0.023). Concurrent TT/IT given before SRS did not gain prognostic significance for 1-year LC (91.4 and 100\% for concurrent TT/IT before SRS and SRS alone, $p=0.197$ ). On further univariate analysis, Melanoma-molGPA was associated with significantly improved LC (Fig. 2b). Gender, BRAF status, PTV margin, V10 and V12 did not gain prognostic significance for LC (Table 3).

\section{Distant intracranial control and overall survival}

Distant intracranial failure was found in 15 of the 28 patients $(53.6 \%)$. Median time to distant intracranial failure was 16 months after first SRS treatment. One- and 2- year DIC rates were 54.2 and $36.6 \%$, respectively (Fig. 1b). DIC rates after 1 year were $47.7 \%$ und $50 \%$ for SRS with concurrent TT/IT and SRS alone $(p=0.933)$. Due to new intracranial metastases, WBRT was applied in 3 patients and 12 patients underwent additional SRS for new metastases. A higher number of brain metastases at first diagnosis (> 3 metastases) was associated with significantly worse distant intracranial control $(p=0.011)$ in univariate analysis. Gender, BRAF status, MelanomamolGPA, and concurrent TT/IT were not of prognostic significance for distant intracranial control (Table 4).

Median OS was 22 months after first diagnosis of brain metastasis. One- and two-year-OS rates were 66.3 and 48.6\% after first diagnosis of brain metastasis (Fig. 1c). Patients with better Melanoma-molGPA-score showed a tendency for better OS $(p=0.087)$. Other factors (gender, BRAF status, number of brain metastases, and concurrent TT/IT) did not gain prognostic significance for OS (Table 4).

\section{Radiation necrosis (RN)}

Symptomatic radiation necrosis was found in 7 lesions (13.5\%). All patients underwent metabolic FET-PET imaging and were treated with steroids or bevacizumab in case of steroid refractory symptoms or steroid induced side effects. Radiation necrosis-free survival (RNFS) rates after one and 2 years were 87.9 and $81.7 \%$, respectively (Fig. 3a). The estimated 1-year RNFS rates were 90.0 and $82.1 \%$ for SRS with concurrent TT/IT and SRS alone $(p=0.935)$ (Fig. 3b). We analyzed concurrent TT/ IT given before and after SRS, neither of these factors are significant for radiation necrosis development ( $p=$ 0.723 and $p=0.935)$. The timing of targeted therapy given before SRS was further analyzed from 7 days until 2 days before SRS, but was not prognostic for brain necrosis.

On further univariate analysis, the volume of normal brain tissue which received $\geq 10$ Gy and $\geq 12$ Gy (V10 and V12) was significantly associated with the occurrence of radiation necrosis $(p<0.001$ and $p=0.004)$ (Table 3).

\section{Discussion}

In our analysis of SRS combined with TT/IT in MBM, we reported several findings: (i) LC was significantly improved in the cohort of SRS and concurrent TT/IT, (ii) distant intracranial control and OS were not significantly different in SRS with concurrent TT/IT and SRS alone, 
Table $\mathbf{2}$ Lesion characteristics and treatment parameters

\begin{tabular}{|c|c|c|}
\hline \multirow{2}{*}{$\begin{array}{l}\text { Parameter } \\
\text { Number of lesions with follow up } \geq 3 \text { months }\end{array}$} & \multicolumn{2}{|c|}{ Number of lesions absolute relative } \\
\hline & 52 & $100 \%$ \\
\hline \multicolumn{3}{|l|}{ Location of metastasis } \\
\hline Left & 26 & $50.0 \%$ \\
\hline Right & 25 & $48.1 \%$ \\
\hline Midline & 1 & $1.9 \%$ \\
\hline Frontal & 19 & $36.5 \%$ \\
\hline Parietal & 6 & $11.5 \%$ \\
\hline Temporal & 8 & $15.4 \%$ \\
\hline Occipital & 6 & $11.5 \%$ \\
\hline Frontotemporal & 1 & $1.9 \%$ \\
\hline Temporoparietal & 1 & $1.9 \%$ \\
\hline Parietooccipital & 1 & $1.9 \%$ \\
\hline Cerebellum & 3 & $5.8 \%$ \\
\hline Others (basal ganglia, insular cortex, parahippocampal, parafalcin) & 7 & $13.5 \%$ \\
\hline \multicolumn{3}{|l|}{ GTV to PTV margin (mm) } \\
\hline $2 \mathrm{~mm}$ & 24 & $46.2 \%$ \\
\hline $3 \mathrm{~mm}$ & 28 & $53.8 \%$ \\
\hline \multicolumn{3}{|l|}{ Prescribed dose (Gy) } \\
\hline 15 & 1 & $1.9 \%$ \\
\hline 17 & 2 & $3.8 \%$ \\
\hline 18 & 17 & $32.7 \%$ \\
\hline 20 & 30 & $57.7 \%$ \\
\hline 24 & 2 & $3.8 \%$ \\
\hline \multicolumn{3}{|l|}{ Prescribed isodose (\%) } \\
\hline 70 & 1 & $1.9 \%$ \\
\hline 80 & 51 & $98.1 \%$ \\
\hline \multicolumn{3}{|l|}{ Treatment planning software } \\
\hline Oncentra Masterplan & 30 & $57.7 \%$ \\
\hline Monaco & 17 & $32.7 \%$ \\
\hline Radionics & 5 & $9.6 \%$ \\
\hline \multicolumn{3}{|l|}{ Fixation technique } \\
\hline Frameless Brainlab mask & 9 & $17.3 \%$ \\
\hline Frameless double thermoplastic mask & 38 & $73.1 \%$ \\
\hline Invasive fixed head frame & 5 & $9.6 \%$ \\
\hline \multicolumn{3}{|l|}{ Targeted/ immunotherapy (TT/IT) } \\
\hline BRAFi & 5 & $9.6 \%$ \\
\hline MEKi & 1 & $1.9 \%$ \\
\hline Anti-CTLA-4 & 4 & $7.7 \%$ \\
\hline Anti-PD1 & 14 & $26.9 \%$ \\
\hline BRAFi+MEKi & 6 & $11.5 \%$ \\
\hline Anti-PD1 + Anti-CTLA-4 & 10 & $19.2 \%$ \\
\hline Triple combination & 2 & $3.8 \%$ \\
\hline none & 10 & $19.2 \%$ \\
\hline
\end{tabular}

Application of TT/IT 
Table 2 Lesion characteristics and treatment parameters (Continued)

\begin{tabular}{lll}
\hline Parameter & \multicolumn{2}{l}{ Number of lesions absolute relative } \\
\cline { 2 - 3 } Number of lesions with follow up $\geq 3$ months & 52 & $100 \%$ \\
\hline Before SRS (median: 9.5 days, range: 3-43 days) & 24 & $46.2 \%$ \\
Concurrent before SRS (median: 8 days, range: 3-21 days) & 21 & $40.4 \%$ \\
After SRS (median: 9 days, range: 2-97 days) & 39 & $75.0 \%$ \\
Concurrent after SRS (median: 8 days, range: 2-27 days) & 15 & $28.8 \%$ \\
Concurrent (4 weeks before or 4 weeks after SRS) & 36 & $69.2 \%$ \\
\hline
\end{tabular}

(iii) radiation necrosis rate was not significantly different in the two groups.

We reported an excellent 1-year local control rate of 98.1\% and local failure has been found in only two lesions. TT/IT concurrent with SRS revealed a statistically significant better LC than non-concurrent TT/IT /SRS alone (one-year LC $100 \%$ versus $83 \% ; p=0.023$ ). This finding is in accordance with preceding studies, which showed a statistically significant difference in LC favoring concurrent therapy [17-19]. Our analysis also confirmed that Melanoma-molGPA, which incorporated BRAF mutation status into its calculation, is a prognostic factor for LC. Similar results have been shown in previous studies [12, 20].

After the diagnosis of brain metastases, our cohort showed 1-year OS rates of $66.3 \%$ in both groups, which is in accordance with other analyses [17, 21-23]. A significant OS difference, with OS improvement in SRS and concurrent TT/IT has been described in other analyses $[5,17,19,20,24]$. Our analysis showed a one-year-OS rate of $68.2 \%$ in the group concurrent TT/IT and SRS and of $50 \%$ in the sequential TT/IT /SRS alone group. However, we could not find a significant difference in OS between these two groups, probably because of the small numbers of patients in our analysis.

We found 1- and 2-year distant intracranial control rates of 54.4 and $36.6 \%$, which were comparable to and slightly better than other reports [25]. However, we were not able to show a significant difference in DIC between the sequential TT/IT /SRS alone group and the concurrent TT/IT group assumably because 4 patients (14.3\%) received WBRT in addition to SRS and due to the limited number of patients included in our analysis.

Regarding the treatment sequencing, previous studies suggested that TT/IT should be administered within 4 weeks of SRS, as this was associated with better intracranial control and survival compared to SRS alone or nonconcurrent TT/IT $[5,26]$. In an analysis from Minniti et al., nivolumab or ipilimumab were administered with a median interval of 3 days from SRS [27]. This study showed that a concurrent SRS with nivolumab or ipilimumab had a meaningful intracranial activity, with an 1-year intracranial PFS rate of $42 \%$ with nivolumab and of $17 \%$ with ipilimumab. Minniti et al. also reported that, a subset of patients (15\%) developed a symptomatic radiation necrosis. With a median follow up of 14 months, symptomatic RN was found in 7 patients (13.5\%) in our cohort. This RN rate is comparable with the aforementioned study. There were no significant differences regarding RN rate between the SRS alone/sequential TT/ IT group and the SRS with concurrent TT/IT group in our analysis. RNFS in patients treated with SRS with concurrent TT/IT was comparable to an historically reported rate of RNFS in SRS alone cohorts [28, 29]. Kohutek et al. reported an actuarial incidence of radiation necrosis of $17.2 \%$ at 12 months after SRS alone [28] and Minniti et al. reported that brain necrosis occurred in $24 \%$ of treated lesions after SRS alone [29]. Nevertheless, it is worth mentioning, that a wider PTV margin (2-3 mm) was applied in our SRS cohort. There was no statistical significant difference regarding brain necrosis between a $2 \mathrm{~mm}$ and a $3 \mathrm{~mm}$ margin in our

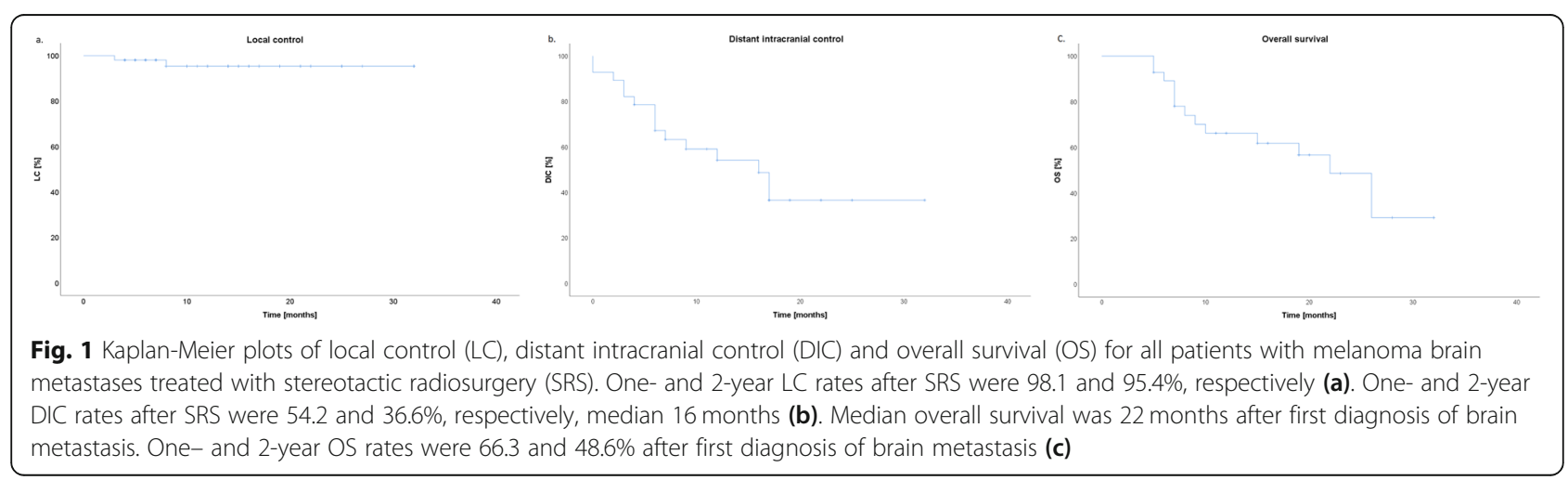




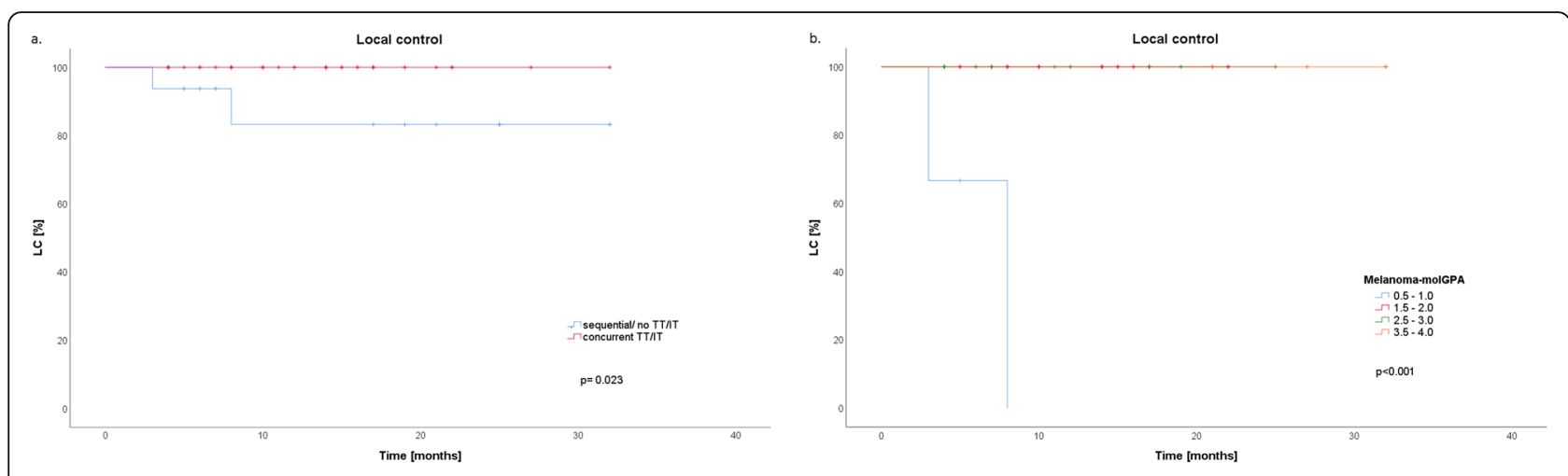

Fig. 2 On univariate analysis, the 1-year local control (LC) rates for stereotactic radiosurgery (SRS) with concurrent targeted/ immunotherapy (TT/ IT) and SRS alone were 100 and 83.3\% ( $p=0.023)$ (a). Melanoma-molGPA as another prognostic factor was also associated with improved LC $(p<0.001)(\mathbf{b})$

Table 3 Univariate analyses of local control (LC) and radiation necrosis-free survival (RNFS) of the 52 lesions

\begin{tabular}{|c|c|c|c|c|c|}
\hline Variable & Lesions (n) & $1 \mathrm{yr}-\mathrm{LC}(\%)$ & $p$-value & $1 \mathrm{yr}-\mathrm{RNFS}(\%)$ & $p$-value \\
\hline \multicolumn{6}{|l|}{ Gender } \\
\hline Male & 33 & 95.7 & & 84.3 & \\
\hline Female & 19 & 94.7 & 0.688 & 94.4 & 0.165 \\
\hline \multicolumn{6}{|l|}{ PTV margin } \\
\hline $2 \mathrm{~mm}$ & 24 & 100 & & 86.3 & \\
\hline $3 \mathrm{~mm}$ & 28 & 90.8 & 0.688 & 90.0 & 0.407 \\
\hline \multicolumn{6}{|c|}{ Targeted/immunotherapy (TT/IT) } \\
\hline Concurrent & 36 & 100 & & 90.0 & \\
\hline No/ sequential & 16 & 83.3 & 0.023 & 82.1 & 0.935 \\
\hline \multicolumn{6}{|c|}{ Concurrent TT/IT before SRS } \\
\hline Yes & 21 & 91.4 & & 78.3 & \\
\hline No & 31 & 100 & 0.197 & 86.9 & 0.723 \\
\hline \multicolumn{6}{|c|}{ Concurrent TT/IT after SRS } \\
\hline Yes & 36 & 100 & & 90.0 & \\
\hline No & 16 & 83.3 & 0.023 & 82.1 & 0.935 \\
\hline \multicolumn{6}{|l|}{ BRAF } \\
\hline BRAF wild type & 27 & 91.5 & & 91.6 & \\
\hline BRAF mutation & 25 & 100 & 0.189 & 81.9 & 0.151 \\
\hline \multicolumn{6}{|l|}{ Melanoma-molGPA } \\
\hline $0.5-1.0$ & 3 & 0 & & 50.0 & \\
\hline $1.5-2.0$ & 27 & 100 & & 90.9 & \\
\hline $2.5-3.0$ & 16 & 100 & & 91.7 & \\
\hline $3.5-4.0$ & 6 & 100 & $<0.001$ & 83.3 & 0.147 \\
\hline \multicolumn{6}{|l|}{ V10 } \\
\hline$\leq 12 \mathrm{ccm}$ & 40 & 94.6 & & 94.4 & \\
\hline$>12 \mathrm{ccm}$ & 12 & 100 & 0.55 & 37.0 & $<0.001$ \\
\hline \multicolumn{6}{|l|}{ V12 } \\
\hline$\leq 10 \mathrm{ccm}$ & 41 & 94.8 & & 91.7 & \\
\hline$>10 \mathrm{ccm}$ & 11 & 100 & 0.587 & 70.0 & 0.004 \\
\hline
\end{tabular}


Table 4 Univariate analyses of distant intracranial control (DIC) and overall survival (OS) of the 28 patients

\begin{tabular}{|c|c|c|c|c|c|}
\hline Variable & Patients $(n=)$ & 1 yr-DIC (\%) & $p$-value & 1 yr-OS (\%) & $p$-value \\
\hline \multicolumn{6}{|l|}{ Gender } \\
\hline Male & 17 & 49.2 & & 61.2 & \\
\hline Female & 11 & 60.6 & 0.216 & 72.7 & 0.426 \\
\hline \multicolumn{6}{|l|}{ BRAF status } \\
\hline BRAF V600-E-Mutation & 14 & 40.1 & & 63.5 & \\
\hline No BRAF V600-E-Mutation & 14 & 54.4 & 0.297 & 69.6 & 0.382 \\
\hline \multicolumn{6}{|l|}{ Melanoma-molGPA } \\
\hline $0.5-1.0$ & 2 & 50.0 & & 0 & \\
\hline $1.5-2.0$ & 11 & 32.7 & & 14.5 & \\
\hline $2.5-3.0$ & 11 & 49.7 & & 53.0 & \\
\hline $3.5-4.0$ & 4 & 50.0 & 0.535 & 75.0 & 0.087 \\
\hline \multicolumn{6}{|l|}{ Number of brain metastases } \\
\hline 1 & 12 & 47.6 & & 72.7 & \\
\hline 2 & 7 & 53.6 & & 57.1 & \\
\hline 3 & 6 & 62.5 & & 50.0 & \\
\hline$>3$ & 3 & 0 & 0.011 & 0 & 0.629 \\
\hline \multicolumn{6}{|l|}{ Targeted/ immunotherapy } \\
\hline Concurrent & 19 & 47.7 & & 64.8 & \\
\hline $\mathrm{No} /$ sequential & 9 & 50.0 & 0.933 & 55.6 & 0.233 \\
\hline
\end{tabular}

analysis. However, Kirkpatrick et al. revealed that radiation necrosis was more frequently seen in the $3-\mathrm{mm}$ arm instead of the 1-mm arm with a low rate of local recurrence in both arms [30]. Therefore, we adopted the 1-mm expansion of the PTV for SRS at our institution after 2017.

We were not able to detect differences in RNFS between SRS with concurrent BRAFi/MEKi and other immunotherapies such as pembrolizumab or nivolumab due to the small number of patients and the heterogeneity of systemic therapies. However, an international multicenter retrospective study did report that the incidence of RN was significantly higher in patients treated with SRS and concurrent BRAFi/MEKi than in patients who underwent SRS and other concurrent immunotherapies [31].

Even though the risk of RN after concurrent therapy is similar to that observed with SRS alone, still the possibility of developing $\mathrm{RN}$ is higher than treating asymptomatic MBM with TT/IT alone. Therefore, it raises important questions about the optimal treatment strategy and sequencing. As the management paradigm of MBM is rapidly changing, preceding studies had analyzed the use of systemic therapy alone as an initial

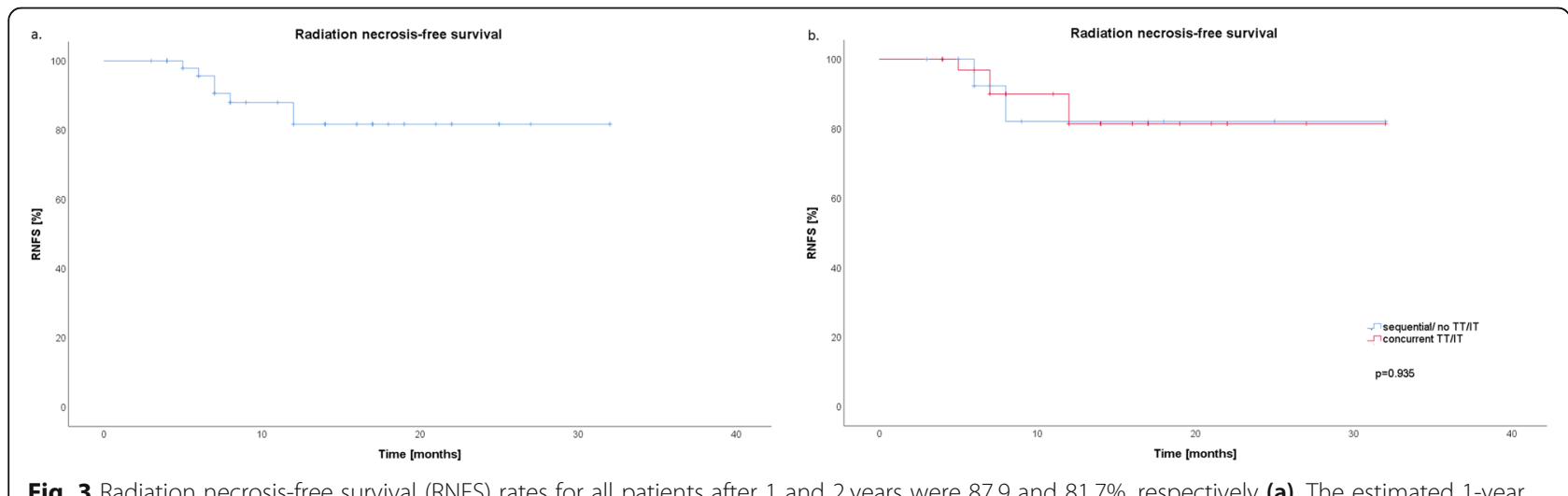

Fig. 3 Radiation necrosis-free survival (RNFS) rates for all patients after 1 and 2 years were 87.9 and $81.7 \%$, respectively (a). The estimated 1-year RNFS rates were 90.0 and $82.1 \%$ for stereotactic radiosurgery (SRS) with concurrent targeted/ immunotherapy (TT/IT) and SRS alone ( $p=0.935)$ (b) 
approach for asymptomatic brain metastases. An open label phase 2 trial reported that ipilimumab showed intracranial activity in some patients with MBM, particularly if metastases were small and asymptomatic. The CNS disease control rate was $10-25 \%$ and the CNS objective response rate was $5-16 \%$. The median duration of intracranial PFS of the brain with ipilimumab alone was 1.2-1.9 months [32]. Another phase 2 multicenter randomized phase 2 study investigated the combination of nivolumab and ipilimumab or nivolumab alone in MBM. The combination of nivolumab and ipilimumab showed a better intracranial response than nivolumab alone in patients with asymptomatic MBM (46 and $20 \%$ ). Median duration of intracranial PFS was not reached in the nivolumab and ipilimumab combination arm and 2.5 months in the nivolumab alone arm [33]. COMBI-MB, an open label multicenter phase 2 trial, analyzed dabrafenib and trametinib in patients with MBM and $\mathrm{BRAF}^{\mathrm{V} 600}$-mutation. Patients with $\mathrm{BRAF}^{\mathrm{V} 600 \mathrm{E}}$-positive, asymptomatic melanoma brain metastases, no previous local brain therapy, and good performance status (ECOG 0 or 1) had an intracranial response rate of $58 \%$ and a response duration of 6.5 months [8]. These studies showed that TT/IT alone had an effective intracranial activity in patients with MBM. Therefore, it is suggested, that TT/IT alone should be considered as first line therapy in patients with asymptomatic MBM and SRS should be performed in case of progression and symptomatic MBM. This approach might require closer patients' follow up and more frequent brain MRI. Nevertheless, since TT/IT alone had overall a short duration of intracranial response and as larger tumor volume is a significant predictor for local control after SRS, postponing SRS might compromise the excellent local control rates of SRS [34]. Besides, the risk of RN also increases with the increasing tumor volume and with the consequently rising PTV, V10 and V12 volume [28, 29]. The latest study reported that multiple brain metastases (up to 10 metastases and maximum diameter of $2.5 \mathrm{~cm}$ ) could be treated in single fraction SRS, which offered an option not just to defer WBRT but also to start TT/IT expeditiously in patients with metastatic melanoma $[35,36]$.

The limitation of our study is its retrospective characters with small numbers of patients including a heterogeneous patient population. Furthermore, toxicity and follow up data are retrospectively collected from chart review, which limits toxicity analysis. These results warrant prospective evaluation of potential synergistic effects and proper timing between TT/IT with SRS to improve outcomes in patients with MBMs.

\section{Conclusion}

SRS with simultaneous TT/IT was well tolerated. The therapy combination significantly improved LC with no difference in radiation necrosis rate. However, prospective studies on SRS with simultaneous TT/IT are necessary to answer open questions of optimal timing and substances of TT/IT and SRS in combination.

\section{Abbreviations}

BM: Brain metastasis; CT: Computed tomography; DIC: Distant intracranial control; FET-PET: 18F-Fluoro-Ethyl-Tyrosinepositron-emission tomography; FFF: Flattening-filter-free; GPA: Graded prognostic assessment; KPS: Karnofsky performance score; LC: Local control; LINAC: Linear accelerator; MBM: Melanoma brain metastasis; MRI: Magnetic resonance imaging; OS: Overall survival; RECIST: Response evaluation criteria in solid tumors; RN: Radiation necrosis; RNFS: radiation necrosis-free survival; SRS: Stereotactic radiosurgery; TT/IT: Targeted/ immunotherapy; WBRT: Whole brain radiotherapy

\section{Acknowledgements}

Not applicable.

\section{Authors' contributions}

$\amalg \mathrm{H}, \mathrm{MN}, \mathrm{CB}, \mathrm{SN}$ design of the work. $\mathrm{H}, \mathrm{OR}$, SN the acquisition, analysis. $\mathrm{H}, \mathrm{OR}$, $\mathrm{RB}, J \mathrm{H}, \mathrm{MN}, \mathrm{CB}, \mathrm{SN}$ interpretation of data, have drafted the work or substantively revised it, have approved the submitted version, have agreed both to be personally accountable for the author's own contributions and to ensure that questions related to the accuracy or integrity of any part of the work.

Funding

Not applicable.

Availability of data and materials

The datasets used and analysed during the current study are available from the corresponding author on reasonable request.

Ethics approval and consent to participate

The institutional review board approved this analysis on 10th of February 2015 and all patients signed informed consent (UE nr. 128-14).

Consent for publication

Not applicable.

\section{Competing interests}

The authors declare that they have no competing interests.

\section{Author details}

'Department of Radiation Oncology, University Hospital, LMU Munich, Munich, Germany. ${ }^{2}$ German Cancer Consortium DKTK, Munich, Germany.

Received: 17 December 2019 Accepted: 4 February 2020

Published online: 14 February 2020

\section{References}

1. Cagney DN, Martin AM, Catalano PJ, Redig AJ, Lin NU, Lee EQ, et al. Incidence and prognosis of patients with brain metastases at diagnosis of systemic malignancy: a population-based study. Neuro Oncol Oxford Univ Press. 2017;19:1511-21.

2. Sampson JH, Carter JH, Friedman AH, Seigler HF. Demographics, prognosis, and therapy in 702 patients with brain metastases from malignant melanoma. J Neurosurg. 2009;88:11-20.

3. Glitza IC, Heimberger AB, Sulman EP, Davies MA. Prognostic factors for survival in melanoma patients with brain metastases. Brain Metastases Prim Tumors Epidemiol Biol Ther Melanoma Other Cancers. 2016:3:26797.

4. Chang EL, Wefel JS, Hess KR, Allen PK, Lang FF, Kornguth DG, et al. Neurocognition in patients with brain metastases treated with radiosurgery or radiosurgery plus whole-brain irradiation: a randomised controlled trial. Lancet Oncol. 2009;10:1037-44.

5. Qian JM, YU JB, Kluger HM, Chiang VLS. Timing and type of immune checkpoint therapy affect the early radiographic response of melanoma 
brain metastases to stereotactic radiosurgery. Cancer U S. 2016;122: 3051-8.

6. Giaj Levra N, Sicignano G, Fiorentino A, Fersino S, Ricchetti F, Mazzola R, et al. Whole brain radiotherapy with hippocampal avoidance and simultaneous integrated boost for brain metastases: a dosimetric volumetric-modulated arc therapy study. Radiol Medica Springer Milan. 2016;121:60-9

7. Gondi V, Pugh SL, Tome WA, Caine C, Corn B, Kanner A, et al. Preservation of memory with conformal avoidance of the hippocampal neural stem-cell compartment during whole-brain radiotherapy for brain metastases (RTOG 0933): a phase II multi-institutional trial. J Clin Oncol. 2014;32:3810-6.

8. Davies MA, Saiag P, Robert C, Grob JJ, Flaherty KT, Arance A, et al. Dabrafenib plus trametinib in patients with BRAFV600-mutant melanoma brain metastases (COMBI-MB): a multicentre, multicohort, open-label, phase 2 trial. Lancet Oncol. 2017.

9. Lehrer EJ, Peterson J, Brown PD, Sheehan JP, Quiñones-Hinojosa A, Zaorsky NG, et al. Treatment of brain metastases with stereotactic radiosurgery and immune checkpoint inhibitors: an international metaanalysis of individual patient data. Radiother Oncol [internet]. Elsevier B. V.; 2019;130:104-12. Available from: https://doi.org/https://doi.org/10. 1016/j.radonc.2018.08.025.

10. Flanigan JC, Jilaveanu LB, Faries M, Sznol M, Ariyan S, Yu JB, et al. Melanoma Brain Metastases. Time Reassess Bias ? 2012:35:200-10.

11. Markovic SN. Sarkaria JN. Elmquist WF metastases. 2017;2:309-25.

12. Sperduto PW, Jiang W, Brown PD, Braunstein S, Sneed P, Wattson DA, et al. Estimating Survival in Melanoma Patients With Brain Metastases: An Update of the Graded Prognostic Assessment for Melanoma Using Molecular Markers (Melanoma-molGPA). Int J Radiat Oncol Biol Phys. Elsevier Inc. 2017;99:812-6.

13. Baumert BG, Rutten I, Dehing-Oberije C, Twijnstra A, Dirx MJM, Debougnoux-Huppertz RMTL, et al. A pathology-based substrate for target definition in radiosurgery of brain metastases. Int J Radiat Oncol Biol Phys. 2006;66:187-94.

14. Eisenhauer EA, Therasse P, Bogaerts J, Schwartz LH, Sargent D, Ford R, et al. New response evaluation criteria in solid tumours: Revised RECIST guideline (version 1.1). Eur J Cancer. 2009;45:228-47.

15. Detsky JS, Keith J, Conklin J, Symons S, Myrehaug S, Sahgal A, et al. Differentiating radiation necrosis from tumor progression in brain metastases treated with stereotactic radiotherapy: utility of intravoxel incoherent motion perfusion MRI and correlation with histopathology. J Neurooncol Springer US. 2017;134:433-41

16. Bodensohn R, Hadi I, Fleischmann DF, Corradini S, Thon N, Rauch J, et al Bevacizumab as a treatment option for radiation necrosis after cranial radiation therapy: a retrospective monocentric analysis. Strahlentherapie und Onkol. 2019.

17. Kiess AP, Wolchok JD, Barker CA, Postow MA, Tabar V, Huse JT, et al. Stereotactic radiosurgery for melanoma brain metastases in patients receiving ipilimumab: Safety profile and efficacy of combined treatment. Int J Radiat Oncol Biol Phys [Internet]. Elsevier Inc.; 2015;92:368-375. Available from: http://dx.doi.org/https://doi.org/10.1016/j.ijrobp.2015.01. 004

18. Yusuf MB, Amsbaugh MJ, Burton E, Chesney J, Woo S. Peri-SRS Administration of Immune Checkpoint Therapy for Melanoma Metastatic to the Brain: Investigating Efficacy and the Effects of Relative Treatment Timing on Lesion Response. World Neurosurg [Internet]. Elsevier Inc; 2017;100:632640.e4. Available from: http://dx.doi.org/https://doi.org/10.1016/j.wneu.2017. 01.101

19. Cohen-Inbar O. PS1-140 The Effect of Timing of Stereotactic Radiosurgery Treatment of Melanoma Brain Metastases Treated with Ipilimumab. Can J Neurol Sci / J Can des Sci Neurol. 2016;43:S6-7.

20. Rauschenberg R, Bruns J, Bru J, Daubner D, Lohaus F, Zimmer L, et al. Impact of radiation, systemic therapy and treatment sequencing on survival of patients with melanoma brain metastases. Eur J Cancer. 2019;110:11-20.

21. Choong ES, Lo S, Drummond M, Fogarty GB, Menzies AM, Guminski A, et al. Survival of patients with melanoma brain metastasis treated with stereotactic radiosurgery and active systemic drug therapies. Eur J Cancer [internet]. Elsevier Ltd; 2017;75:169-178. Available from: http://dx. doi.org/https://doi.org/10.1016/j.ejca.2017.01.007

22. Gaudy-Marqueste C, Dussouil AS, Carron R, Troin L, Malissen N, Loundou A, et al. Survival of melanoma patients treated with targeted therapy and immunotherapy after systematic upfront control of brain metastases by radiosurgery. Eur J Cancer. 2017;84:44-54.

23. Acharya S, Mahmood M, Mullen D, Yang D, Tsien Cl, Huang J, et al. Distant intracranial failure in melanoma brain metastases treated with stereotactic radiosurgery in the era of immunotherapy and targeted agents. Adv Radiat Oncol [internet]. Elsevier Inc.; 2017;2:572-580. Available from: https://doi.org/ https://doi.org/10.1016/j.adro.2017.07.003.

24. Chen L, Douglass J, Kleinberg L, Ye X, Marciscano AE, Forde PM, et al. Concurrent immune checkpoint inhibitors and stereotactic radiosurgery for brain metastases in non-small cell lung Cancer, melanoma, and renal cell carcinoma. Int J Radiat Oncol biol Phys [internet]. Elsevier Inc.; 2018;100: 916-925. Available from: https://doi.org/https://doi.org/10.1016/j.jjrobp.2017. 11.041.

25. Ahmed KA, Stallworth DG, Kim Y, Johnstone PAS, Harrison LB, Caudell J, et al. Clinical outcomes of melanoma brain metastases treated with stereotactic radiation and anti-PD-1 therapy. Ann Oncol. 2016;27:434-41.

26. Patel KR, Shoukat S, Oliver DE, Chowdhary M, Rizzo M, Lawson DH, et al. Ipilimumab and stereotactic radiosurgery versus stereotactic radiosurgery alone for newly diagnosed melanoma brain metastases. Am J Clin Oncol Cancer Clin Trials. 2017:40:444-50.

27. Minniti G, Anzellini D, Reverberi C, Cappellini GCA, Marchetti L, Bianciardi F, et al. Stereotactic radiosurgery combined with nivolumab or Ipilimumab for patients with melanoma brain metastases: Evaluation of brain control and toxicity. J Immunother Cancer. J ImmunoTherapy Cancer. 2019;7:1-11.

28. Kohutek ZA, Yamada Y, Chan TA, Brennan CW, Tabar V, Gutin PH, et al. Long-term risk of radionecrosis and imaging changes after stereotactic radiosurgery for brain metastases. J Neurooncol. 2015;125:149-56. Available from: http://www.ncbi.nlm.nih.gov/pubmed/26307446. Accessed 17 Jan 2020.

29. Minniti G, Clarke E, Lanzetta G, Osti MF, Trasimeni G, Bozzao A, et al. Stereotactic radiosurgery for brain metastases: analysis of outcome and risk of brain radionecrosis. Radiat Oncol [Internet]. 2011;6:48. Available from: http://www.pubmedcentral.nih.gov/articlerender.fcgi?artid=31083 08\&tool=pmcentrez\&rendertype=abstract. Accessed 17 Jan 2020.

30. Kirkpatrick JP, Wang Z, Sampson JH, McSherry F, Herndon JE, Allen KJ, et al. Defining the optimal planning target volume in image-guided stereotactic radiosurgery of brain metastases: results of a randomized trial. Int J Radiat Oncol Biol Phys. Elsevier Inc.; 2015;91:100-8. Available from: http://linkinghub.elsevier.com/retrieve/pii/S0360301614040887\%5Cn, http:// www.ncbi.nlm.nih.gov/pubmed/25442342. Accessed 17 Jan 2020.

31. Kroeze SGC, Fritz C, Hoyer M, Lo SS, Ricardi U, Sahgal A, et al. Toxicity of concurrent stereotactic radiotherapy and targeted therapy or immunotherapy: A systematic review. Cancer Treat Rev [Internet]. Author(s); 2017;53:25-37. Available from: http://dx.doi.org/https://doi.org/ 10.1016/j.ctrv.2016.11.013

32. Margolin K, Ernstoff MS, Hamid O, Lawrence D, McDermott D, Puzanov I, et al. Ipilimumab in patients with melanoma and brain metastases: an open-label, phase 2 trial. Lancet Oncol [internet]. Elsevier Itd. 2012;13:459-65 Available from: http://www.ncbi.nlm.nih.gov/pubmed/22456429.

33. Long G V., Atkinson V, Lo S, Sandhu S, Guminski AD, Brown MP, et al. Combination nivolumab and ipilimumab or nivolumab alone in melanoma brain metastases: a multicentre randomised phase 2 study. Lancet Oncol. 2018;19:672-81

34. Garsa AA, Badiyan SN, Dewees T, Simpson JR, Huang J, Drzymala RE, et al. Predictors of individual tumor local control after stereotactic radiosurgery for non-small cell lung cancer brain metastases. Int J Radiat Oncol Biol Phys. Elsevier Inc. 2014:90:407-13.

35. Hofmaier J, Bodensohn R, Garny S, Hadi I, Fleischmann DF, Eder M, et al. Single isocenter stereotactic radiosurgery for patients with multiple brain metastases: Dosimetric comparison of VMAT and a dedicated DCAT planning tool. Radiat Oncol Radiat Oncol. 2019;14:4-11.

36. Ruggieri R, Naccarato S, Mazzola R, Ricchetti F, Corradini S, Fiorentino A, et al. Linac-based radiosurgery for multiple brain metastases: Comparison between two mono-isocenter techniques with multiple non-coplanar arcs. Radiother Oncol. Elsevier B.V. 2019;132:70-8.

\section{Publisher's Note}

Springer Nature remains neutral with regard to jurisdictional claims in published maps and institutional affiliations. 